International Mathematical Forum, 1, 2006, no. 4, 157 - 163

\title{
CHARACTERIZATION OF THE EXISTENCE OF A WEAK QUALITATIVE PROBABILITY
}

\author{
Gianni BOSI \\ Dipartimento di Matematica Applicata \\ "Bruno de Finetti", Università di Trieste, \\ Piazzale Europa 1, \\ 34127 Trieste, Italy
}

\begin{abstract}
Necessary and sufficient conditions are presented for the existence of a weak qualitative probability for a binary relation on an algebra of subsets of an arbitrary set.
\end{abstract}

Mathematics Subject Classification: 60A05, 91B06.

Keywords: Qualitative probability, weak utility.

\section{Introduction}

The problem concerning the representability of a total preorder (i.e., a reflexive, transitive and total binary relation) on an algebra of subsets of a given nonempty set by means of a probability measure was already solved by several authors (see e.g. Chateauneuf [4], Clark [5], and Fine [7]). The existence of a weak probability representation was characterized by Cohen [6], who followed a dual approach. Nakamura [10] was concerned with the representability of a binary relation with intransitive indifference by means of a pair of probability measures (see also Fishburn [9]).

On the other hand, the notion of a lower scale in a topological preordered space proved to be useful in order to characterize the existence of a continuous or at least semicontinuous utility representation in case that the preorder is not complete (see e.g. Alcantud and Rodríguez-Palmero [1] and Alcantud et al. [2]). Further, when we consider a preorder on a group, it is possible to characterize the existence of an additive utility function by imposing suitable conditions on a lower scale (see e.g. Bosi et al. [3]).

In this paper, we use the concept of a lower (decreasing) scale in order to obtain a characterization of the existence of a probability measure which is 
also a weak utility for an acyclic binary relation on an algebra of subsets of a nonempty set. To the best of our knowledge, this approach appears as novel in the literature so far. As an application of our main result, we present a sufficient condition for the existence of a probability measure which is a strong utility for a linear order on an algebra of subsets of a nonempty set under the assumption of order separability.

\section{Notation and preliminaries}

Throughout this paper we shall denote by $\prec$ a binary relation on an algebra $\mathcal{A}_{\Omega}$ of subsets of an arbitrary set $\Omega$. As usual, if for two events $A, B \in \mathcal{A}_{\Omega}$ it happens that $A \prec B$, then this means that " $B$ is more probable than $A$ ". The pair $\left(\mathcal{A}_{\Omega}, \prec\right)$ will be referred to as a related algebra.

Definition 2.1 A set function $p: \mathcal{A}_{\Omega} \rightarrow[0,1]$ on a related algebra $\left(\mathcal{A}_{\Omega}, \prec\right)$ is said to be a qualitative probability on $\left(\Omega, \mathcal{A}_{\Omega}, \prec\right)$ if $p$ is both a probability on $\left(\Omega, \mathcal{A}_{\Omega}\right)$ (i.e., $p(\emptyset)=0, p(\Omega)=1$ and $p(A \cup B)=p(A)+p(B)$ for all sets $A, B \in \mathcal{A}_{\Omega}$ such that $\left.A \cap B=\emptyset\right)$ and a weak utility on $\left(\mathcal{A}_{\Omega}, \prec\right)$ (i.e., for all sets $A, B \in \mathcal{A}_{\Omega}, p(A)<p(B)$ whenever $\left.A \prec B\right)$.

If in addition for all sets $A, B \in \mathcal{A}_{\Omega}$ we have that $p(A)<p(B)$ is equivalent to $A \prec B$ (i.e., $p$ is a strong utility on $\left(\mathcal{A}_{\Omega}, \prec\right)$ ), then $p$ will be referred to as a strong qualitative probability.

Remark 2.2 If there exists a weak utility $p$ on $\left(\mathcal{A}_{\Omega}, \prec\right)$, then $\prec$ is acyclic (i.e., $A_{1} \prec A_{2} \prec \ldots \prec A_{n}$ implies $A_{1} \neq A_{n}$ for all $A_{1}, \ldots, A_{n} \in \mathcal{A}_{\Omega}$ ).

Given an acyclic binary relation $\prec$ on $\mathcal{A}_{\Omega}$, we shall denote by $\prec$ the transitive closure of $\prec$ (i.e., $A \prec B$ if and only if there exist $A_{1}, \ldots, A_{n} \in \mathcal{A}_{\Omega}$ such that $A=A_{1} \prec A_{2} \prec \ldots \prec A_{n}=B$ ). The transitive closure of any acyclic binary relation is a partial order (i.e., it is irreflexive and transitive). Obviously, a partial order $\prec$ is in particular acyclic and in this case we have that $\prec=\prec$.

Remark 2.3 If there exists a strong utility $p$ on $\left(\mathcal{A}_{\Omega}, \prec\right)$, then $\prec$ is a weak order (i.e., $\prec$ is asymmetric and negatively transitive).

Definition 2.4 A binary relation $\prec$ on $\mathcal{A}_{\Omega}$ is said to be additive if for all sets $A, B, C \in \mathcal{A}_{\Omega}$ such that $(A \cup B) \cap C=\emptyset$ we have that $A \prec B$ if and only if $A \cup C \prec B \cup C$.

If there exists a weak qualitative probability $p$ on a linearly ordered algebra $\left(\mathcal{A}_{\Omega}, \prec\right)$ (i.e., the binary relation $\prec$ is irreflexive, transitive and complete), then $p$ is also a strong qualitative probability and $\prec$ is necessarily additive. 
Definition 2.5 If $\left(\mathcal{A}_{\Omega}, \prec\right)$ is a related algebra, then a subset $\mathcal{G}$ of $\mathcal{A}_{\Omega}$ is a said to be a lower set if the following condition holds:

$$
A \in \mathcal{G} \text { and } B \prec A \text { imply } B \in \mathcal{G} \text { for all sets } A, B \in \mathcal{A}_{\Omega} \text {. }
$$

Definition 2.6 If $\prec$ is an acyclic binary relation on an algebra $\mathcal{A}_{\Omega}$ of subsets of $\Omega$, then a countable family $\left.\left.\mathcal{G}=\left\{G_{r}: r \in \mathbb{Q} \cap\right] 0,1\right]\right\}$ of lower subsets of $\mathcal{A}_{\Omega}$ is said to be a countable lower scale in $\left(\Omega, \mathcal{A}_{\Omega}, \prec\right)$ if it satisfies the following conditions:

(1) $G_{1}=\mathcal{A}_{\Omega}$ and $G_{r} \subset G_{s}$ for all $\left.\left.r, s \in \mathbb{Q} \cap\right] 0,1\right]$ such that $r<s$;

(2) $\emptyset \in G_{r}$ for all $\left.\left.r \in \mathbb{Q} \cap\right] 0,1\right]$ and $\Omega \notin G_{r}$ for all $\left.r \in \mathbb{Q} \cap\right] 0,1[$.

Further, a countable lower scale $\left.\left.\mathcal{G}=\left\{G_{r}: r \in \mathbb{Q} \cap\right] 0,1\right]\right\}$ in $\left(\Omega, \mathcal{A}_{\Omega}, \prec\right)$ is said to be separating if the following condition holds:

$$
\begin{array}{r}
\text { for all } \left.A, B \in \mathcal{A}_{\Omega} \text { such that } A \prec \prec B \text { there exist } r, s \in \mathbb{Q} \cap\right] 0,1[ \\
\text { such that } r<s, A \in G_{r} \text { and } B \notin G_{s} .
\end{array}
$$

Definition 2.7 If $\prec$ is an acyclic binary relation on an algebra $\mathcal{A}_{\Omega}$ of subsets of $\Omega$, then a countable lower scale $\left.\left.\mathcal{G}=\left\{G_{r}: r \in \mathbb{Q} \cap\right] 0,1\right]\right\}$ in $\left(\Omega, \mathcal{A}_{\Omega}, \prec\right)$ is said to be additive if for all $A, B \in \mathcal{A}_{\Omega}$ such that $A \cap B=\emptyset$, and for all $r, s \in \mathbb{Q} \cap] 0,1[$ the following conditions hold:

$$
\begin{aligned}
& A \cup B \in G_{r+s} \text { whenever } A \in G_{r}, B \in G_{s}, r+s<1 ; \\
& (r+s=1) \text { or }\left(r+s<1, A \cup B \notin G_{r+s}\right) \text { whenever } A \notin G_{r}, B \notin G_{s} .
\end{aligned}
$$

Definition 2.8 If $\prec$ is an acyclic binary relation on an algebra $\mathcal{A}_{\Omega}$ of subsets of $\Omega$, then a countable lower scale $\left.\left.\mathcal{G}=\left\{G_{r}: r \in \mathbb{Q} \cap\right] 0,1\right]\right\}$ in $\left(\Omega, \mathcal{A}_{\Omega}, \prec\right)$ is said to be monotonic if the following condition holds:

(6) for all $A, B \in \mathcal{A}_{\Omega}$ and $\left.\left.r \in \mathbb{Q} \cap\right] 0,1\right], B \in G_{r}$ whenever $B \subset A$ and $A \in G_{r}$.

Definition 2.9 A binary relation $\prec$ on an algebra $\mathcal{A}_{\Omega}$ of subsets of $\Omega$ is said to be order separable if there exists a countable subset $\mathcal{D}$ of $\mathcal{A}_{\Omega}$ such that for all $A, B \in \mathcal{A}_{\Omega}$ with $A \prec B$ there exists $D \in \mathcal{D}$ such that $A \prec D \prec B$. $\mathcal{D}$ is said to be an order dense subset of the related algebra $\left(\mathcal{A}_{\Omega}, \prec\right)$. 


\section{Existence of a weak qualitative probability}

The following theorem provides a characterization of the existence of a weak qualitative probability for a binary relation on an algebra of subsets of a given set.

Theorem 3.1 Let $\prec$ be a binary relation on an algebra $\mathcal{A}_{\Omega}$ of subsets of an arbitrary set $\Omega$. Then the following conditions are equivalent:

(i) There exists a weak qualitative probability $p$ on $\left(\Omega, \mathcal{A}_{\Omega}, \prec\right)$;

(ii) $\prec$ is acyclic and there exists a countable lower scale $\left.\left.\mathcal{G}=\left\{G_{r}: r \in \mathbb{Q} \cap\right] 0,1\right]\right\}$ in $\left(\Omega, \mathcal{A}_{\Omega}, \prec\right)$ which is additive, monotonic and separating.

Proof. Necessity. Assume that there exists a weak qualitative probability $p$ on $\left(\Omega, \mathcal{A}_{\Omega}, \prec\right)$. Then we have just to define $G_{r}=p^{-1}([0, r[)$ for all $r \in \mathbb{Q} \cap] 0,1[$ and $G_{1}=\mathcal{A}_{\Omega}$ in order to easily check that the family $\left.\left.\mathcal{G}=\left\{G_{r}: r \in \mathbb{Q} \cap\right] 0,1\right]\right\}$ is a countable lower scale in $\left(\Omega, \mathcal{A}_{\Omega}, \prec\right)$ which is additive, monotonic and separating. Indeed, it is clear that $\mathcal{G}$ is actually a countable lower scale in $\left(\Omega, \mathcal{A}_{\Omega}, \prec\right)$. If $A$ and $B$ are any two elements of $\mathcal{A}_{\Omega}$ such that $A \prec \prec B$, then $p(A)<p(B)$ since $p$ is in particular a weak utility on the related set $\left(\mathcal{A}_{\Omega}, \prec\right)$, and therefore if we consider any two rational numbers $r, s$ such that $p(A)<r<s<p(B)$ we have that $A \in G_{r}, B \notin G_{s}$. Hence, the countable lower scale $\mathcal{G}$ is also separating according to condition (3). Now consider any two sets $A, B \in \mathcal{A}_{\Omega}$ such that $A \cap B=\emptyset$. If $A \in G_{r}, B \in G_{s}$ for some $\left.\left.r, s \in \mathbb{Q} \cap\right] 0,1\right]$ such that $r+s<1$, then we have that $p(A \cup B)=p(A)+p(B)<r+s$, and therefore $A \cup B \in G_{r+s}$. If $A \notin G_{r}, B \notin G_{s}$ for some $\left.\left.r, s \in \mathbb{Q} \cap\right] 0,1\right]$ then $r+s>1$ is contradictory since this would imply that $p(A)+p(B)=p(A \cup B) \geq r+s>1$. So assume that $r+s<1$. Then we have that $p(A \cup B)=p(A)+p(B) \geq r+s$, which implies that $A \cup B \notin G_{r+s}$. Hence, the above defined countable lower scale $\mathcal{G}$ is additive according to Definition 2.7. Finally, if for some $A, B \in \mathcal{A}_{\Omega}$ such that $B \subset A$, and $r \in \mathbb{Q} \cap] 0,1]$ we have that $A \in G_{r}$, then $p(B) \leq p(A)<r$ obviously implies $B \in G_{r}$. Hence, $\mathcal{G}$ is monotonic according to Definition 2.8. Sufficiency. Assume that there exists a countable lower scale $\left.\left.\mathcal{G}=\left\{G_{r}: r \in \mathbb{Q} \cap\right] 0,1\right]\right\}$ in $\left(\Omega, \mathcal{A}_{\Omega}, \prec\right)$ which is additive, monotonic and separating. Define the function $p: \mathcal{A}_{\Omega} \rightarrow[0,1]$ by

$$
\left.p(A)=\inf \{r \in \mathbb{Q} \cap] 0,1]: A \in G_{r}\right\} \quad\left(A \in \mathcal{A}_{\Omega}\right) .
$$

We claim that $p$ is a weak qualitative probability on $\left(\Omega, \mathcal{A}_{\Omega}, \prec\right)$. Let us first show that $p$ is a weak utility on $\left(\mathcal{A}_{\Omega}, \prec\right)$. Since $\mathcal{G}$ is separating, we have that for every $A, B \in \mathcal{A}_{\Omega}$ such that $A \prec B$ there exist $\left.r, s \in \mathbb{Q} \cap\right] 0,1[$ such that $r<s, A \in G_{r}, B \notin G_{s}$, and therefore from the definition of $p$ we have that 
$p(A) \leq r<s \leq p(B)$, which obviously implies that $p(A)<p(B)$.

Let us now prove that $p$ is a probability measure on $\left(\Omega, \mathcal{A}_{\Omega}\right)$. By condition (2) in the definition of a countable lower scale (see Definition 2.6), it is clear that $p(\emptyset)=0$ and $p(\Omega)=1$. Further, we have that $0 \leq p(A) \leq 1$ directly from the definition of $p$. In order to prove that $p$ is additive, assume that there exist $A, B \in \mathcal{A}_{\Omega}$ such that $A \cap B=\emptyset$ and $p(A \cup B) \neq p(A)+p(B)$. If $p(A)+p(B)<p(A \cup B)$, then both $p(A)$ and $p(B)$ must be strictly less than 1. From the definition of $p$, there exist $r, s \in \mathbb{Q} \cap[0,1]$ such that $p(A)<r$, $p(B)<s, A \in G_{r}, B \in G_{s}$ and $p(A)+p(B)<r+s<p(A \cup B)$. But here we have a contradiction since by condition (4) in the definition of an additive lower scale (see Definition 2.7), we should have that $A \cup B \in G_{r+s}$, which implies $p(A \cup B) \leq r+s$ from the definition of $p$. If $p(A \cup B)<p(A)+p(B)$, then first assume that $p(A)$ and $p(B)$ are both strictly positive. By condition (5) we may assume that $p(A \cup B)<1$ because otherwise there would exist $r, s \in \mathbb{Q} \cap] 0,1[$ such that $r<p(A), s<p(B), r+s>1$, and therefore it should be $A \notin G_{r}, B \notin G_{s}, r+s>1$. So assume that $p(A \cup B)<1$. Then we may consider $r, s \in \mathbb{Q} \cap] 0,1[$ such that $r<p(A), s<p(B), p(A \cup B)<r+s<1$. Hence we have that $A \notin G_{r}, B \notin G_{s}$, and therefore $p(A \cup B)<r+s$ is contradictory by condition (5). Finally, if either $p(A)$ or $p(B)$ is equal to 0 , then $p(A \cup B)<p(A)+p(B)$ is contradictory by axiom (6). So the proof is complete.

Remark 3.2 Obviously, from considerations above, we have that Theorem 3.1 applies in case that $\prec$ is a partial order. Further, the previous theorem substantially provides a characterization of the existence of a weak qualitative probability $p$ on a triplet $\left(\Omega, \mathcal{A}_{\Omega}, \precsim\right)$, where $\precsim$ is a preorder (i.e., a reflexive and transitive binary relation) on $\mathcal{A}_{\Omega}$ and $p$ is now a utility on the preordered set $\left(\mathcal{A}_{\Omega}, \precsim\right)$ (i.e., for all sets $A, B \in \mathcal{A}_{\Omega}, p(A) \leq p(B)$ whenever $A \precsim B$ and $p(A)<p(B)$ whenever $A \prec B$ ). Here, $\prec$ is the strict part of $\precsim$ (i.e., for all sets $A, B \in \mathcal{A}_{\Omega}, A \prec B$ if and only if $(A \precsim B)$ and $\left.\operatorname{not}(B \precsim A)\right)$. We only have to replace the term "lower scale" by the term "decreasing scale" where a decreasing scale is a family $\left.\left.\mathcal{G}=\left\{G_{r}: r \in \mathbb{Q} \cap\right] 0,1\right]\right\}$ which consists of decreasing sets (i.e., subsets $\mathcal{G}$ of $\mathcal{A}_{\Omega}$ such that $A \in \mathcal{G}$ and $B \precsim A$ imply $B \in \mathcal{G}$ for all sets $A, B \in \mathcal{A}_{\Omega}$ ).

As an application of the previous characterization we present a sufficient condition for the existence of a strong qualitative probability on an algebra endowed with an order separable linear order.

Corollary 3.3 Let $\prec$ be a linear order on an algebra $\mathcal{A}_{\Omega}$ of subsets of an arbitrary set $\Omega$. Then there exists a strong qualitative probability $p$ on $\left(\Omega, \mathcal{A}_{\Omega}, \prec\right.$ ) provided that the following conditions hold: 
(a) $\emptyset \prec A \prec \Omega$ for every set $A \in \mathcal{A}_{\Omega}$ such that $A \neq \emptyset$ and $A \neq \Omega$;

(b) $\prec$ is additive;

(c) $\prec$ is order separable and there exists a countable order dense subset $\mathcal{D}=\left\{D_{r}: r \in \mathbb{Q} \cap\right] 0,1[\}$ of $\left(\mathcal{A}_{\Omega}, \prec\right)$ satisfying the following conditions:

(c1) $r<s$ is equivalent to $D_{r} \prec D_{s}$ for all $\left.r, s \in \mathbb{Q} \cap\right] 0,1[$;

(c2) $A \prec D_{r}$ and $B \prec D_{s}$ imply $A \cup B \prec D_{r+s}$ for all $A, B \in \mathcal{A}_{\Omega}$ such that $A \cap B=\emptyset$ and for all $r, s \in \mathbb{Q} \cap] 0,1[$ such that $r+s<1$;

(c3) $\operatorname{not}\left(A \prec D_{r}\right)$ and $\operatorname{not}\left(B \prec D_{s}\right)$ imply $(r+s=1)$ or $(r+s<1$, $\left.\operatorname{not}\left(A \cup B \prec D_{r+s}\right)\right)$ for all $A, B \in \mathcal{A}_{\Omega}$ such that $A \cap B=\emptyset$ and for all $r, s \in \mathbb{Q} \cap] 0,1[$.

Proof. Assume that conditions (a), (b) and (c) hold, and then define, for every $r \in \mathbb{Q} \cap] 0,1\left[, G_{r}=\left\{A \in \mathcal{A}_{\Omega}: A \prec D_{r}\right\}\right.$. Further, let $G_{1}=\mathcal{A}_{\Omega}$ and define $\left.\left.\mathcal{G}=\left\{G_{r}: r \in \mathbb{Q} \cap\right] 0,1\right]\right\}$. By Theorem 3.1 it suffices to show that under our assumptions $\mathcal{G}$ is an additive, monotonic and separating countable lower scale in $\left(\Omega, \mathcal{A}_{\Omega}, \prec\right)$. It is immediate to check that $\mathcal{G}$ is actually a lower scale by conditions (a) and (c1). By conditions (c2) and (c3) it is immediate to verify that $\mathcal{G}$ is additive. In order to show that $\mathcal{G}$ is also separating, consider any two sets $A, B \in \mathcal{A}_{\Omega}$ such that $A \prec B$. Since $\prec$ is order separable and condition (c1) holds, there exist two rational numbers $r<s(r, s \in \mathbb{Q} \cap] 0,1[)$ such that $A \prec D_{r} \prec D_{s} \prec B$, or equivalently $A \in G_{r}, B \notin G_{s}$. It remains to show that $\mathcal{G}$ is monotonic. But under our assumptions we have that the following property holds:

(*) $A \prec B$ for all $A, B \in \mathcal{A}_{\Omega}$ such that $A \varsubsetneqq B$.

Indeed, consider any two sets $A, B \in \mathcal{A}_{\Omega}$ such that $A \varsubsetneqq B$. Then, since $\prec$ is a complete binary relation, we have that either $A \prec B$ or $B \prec A$. But $B \prec A$ is contradictory since in this case additivity of $\prec$ would imply $B \cup(\Omega \backslash B)=\Omega \prec A \cup(\Omega \backslash B)$. Hence, the above property $\left(^{*}\right)$ implies that the countable lower scale $\mathcal{G}$ is also monotonic. This consideration completes the proof.

\section{References}

[1] J.C.R. Alcantud and C. Rodríguez-Palmero, Characterization of the existence of semicontinuous weak utilities, Journal of Mathematical Economics, 32 (1999), 503-509. 
[2] J.C.R. Alcantud, G. Bosi, M.J. Campión, J.C. Candeal, E. Induráin, C. Rodríguez-Palmero and M.E. Zuanon, Continuous utility functions through scales, (2005), submitted.

[3] G. Bosi, M.J. Campión, J.C. Candeal, E. Induráin and M.E. Zuanon, Numerical isotonies of preordered semigroups through the concept of a scale, Mathematica Pannonica, 16 (2005), 65-77.

[4] A. Chateauneuf, On the existence of a probability measure compatible with a total preorder on a Boolean algebra, Journal of Mathematical Economics, 14 (1985), 43-52.

[5] S.A. Clark, The measurement of qualitative probability, Journal of Mathematical Psychology, 44 (2000), 464-479.

[6] M.A. Cohen, Necessary and sufficient conditions for existence and uniqueness of weak probability structures, Journal of Mathematical Psychology, 35 (1991), 242-259.

[7] T. Fine, A note on the existence of quantitative probability, Annals of Mathematical Statistics, 42 (1971), 1182-1186.

[8] P.C. Fishburn, Linear extensions of additive partial orders, Order, 14 (1997/98), 153-169.

[9] P.C. Fishburn, Interval Orders and Interval Graphs, Wiley, New York, 1985.

[10] Y. Nakamura, Comparative probability with additive threshold, IPPS Economic Working paper, 02-02 (2002), University of Tsukuba

Received: June 21, 2005 